\title{
Thoracic posture, shoulder muscle activation patterns and isokinetic strength of semi-professional rugby union players
}

\author{
G Bolton, S J Moss, M Sparks, P C Venter \\ Physical Activity, Sport and Recreation, Faculty of Health Sciences, North-West University, Potchefstroom, South Africa \\ G Bolton, MSc \\ S J Moss, PhD, MBA \\ M Sparks, MSc \\ P C Venter, MB ChB, MMed
}

Corresponding author: S J Moss (hanlie.moss@nwu.ac.za)

Background. Shoulder injuries are the most severe injuries in rugby union players, accounting for almost $20 \%$ of injuries related to the sport and resulting in lost playing hours.

Objective. To profile the thoracic posture, scapular muscle activation patterns and rotator cuff muscle isokinetic strength of semi-professional rugby union players.

Methods. Using the hand-behind-the-neck and -back methods, we manually tested the range of motion (ROM) of the shoulder joints of 91 uninjured semi-professional rugby union players who consented to participate in the study. Profiling and classification of thoracic posture was performed according to the New York Posture Test. Activation patterns of the upper and lower trapezius, serratus anterior and infraspinatus scapular muscles were determined by electromyography. The isokinetic muscle strength of the rotator cuff muscles was determined at $60 \% \mathrm{sec}$ by measuring the concentric and eccentric forces during internal rotation (IR) and external rotation (ER).

Results. Participants presented with non-ideal or unsatisfactory internal (59\%) and external (85\%) rotators of the shoulder. A slightly abnormal or abnormal forward head posture was observed in $55 \%$ of participants, while $68 \%$ had an abnormal shoulder position in the lateral view. The muscle activation sequence of the rotator cuff muscles was: (i) serratus anterior, (ii) lower trapezius, (iii) infraspinatus, and (iv) upper trapezius. The isokinetic ER/IR muscle-strength ratio during concentric muscle contraction was $64 \%$ (standard deviation (SD) \pm 14 ) for the left shoulder and $54 \%$ (SD \pm 10 ) for the right shoulder. The ER/IR ratio for eccentric muscle contraction was $67 \%(S D \pm 12)$ and $61 \%(\mathrm{SD} \pm 9)$ for the left and right shoulders, respectively.

Conclusions. Non-ideal or unsatisfactory flexibility of the external rotators of the shoulder, a forward shoulder posture in the lateral view, and weakness of the external rotators did not result in an abnormal rotator cuff muscle activation pattern in this study. Postural deviations may, however, increase the risk of shoulder injury in rugby union players in the long term, and should be corrected.

S Afr J SM 2013;25(1):12-17. DOI:10.7196/SAJSM.366

Poor posture, scapular dyskinesia, altered scapular muscle recruitment patterns and shoulder-strength weaknesses or imbalances may be associated with shoulder injuries in athletes; ${ }^{[1]}$ however, this has not been proven conclusively for rugby players. Despite the fact that rugby union enjoys increasing worldwide popularity, it has one of the highest reported incidences of injury. ${ }^{[2]}$ The shoulder is the second most common site of injury in the rugby union player, accounting for almost $20 \%$ of injuries related to the sport. ${ }^{[3]}$

Despite correlations between rounded shoulders, severe kyphosis and forward head posture with inter-scapula pain among the general population, ${ }^{[4]}$ similar findings are limited with regard to rugby union players. There have been reports, however, of a relationship between postural deviation and incorrect shoulder kinematics. ${ }^{[5]}$

Knowledge of the patterns of shoulder muscle timing and the functional capabilities of the scapular rotators is vitally important to understanding the behaviour of the joint system, particularly under demanding circumstances such as participation in sport. ${ }^{[6,7]}$ Scapulothoracic dysfunction is often seen in patients with shoulder problems. ${ }^{[1,8]}$ Among swimmers with shoulder injuries, there is significantly increased variability in the timing of activation in the upper and lower part of the trapezius muscle ${ }^{[1]}$ reflecting inconsistent or poorly co-ordinated muscle activation. ${ }^{[1]}$ With regard to rugby players, in a study to define muscle-activation patterns in selected shoulder girdle muscles during a front-on tackle in asymptomatic subjects, ${ }^{[9]}$ a consistently earlier activation of the serratus anterior muscle was observed prior to impact, compared with the pectoralis major, biceps brachii, latissimus dorsi and infraspinatus. ${ }^{[9]}$

A combination of electromyography (EMG) and isokinetic dynamometry could provide information regarding the function of shoulder musculature in sport. ${ }^{[6]}$ It has been suggested that the functional strength of the rotator cuff muscles and the rotator-strength ratio are significant predictors of the likelihood of shoulder injury. 
The unilateral muscle ratio - the antagonist/agonist muscle-strength ratio of the infraspinatus and teres minor muscles v. the subscapularis and supraspinatus muscles on the ipsilateral side - is also believed to be important in isokinetic testing. ${ }^{[10]} \mathrm{A}$ sufficient balance between agonist and antagonist muscle groups apparently provides dynamic stabilisation to the shoulder joint. ${ }^{[10]}$ To provide optimal muscle balance and functional capability for overhead athletes, the strength of the external rotators of the glenohumeral joint should be $65-75 \%$ of that of the internal rotator muscles. ${ }^{[10]}$ Muscle-strength ratios that lie outside the proposed normative ranges may increase the risk of injury to athletes. ${ }^{[11]}$ Furthermore, there is evidence that rugby union players, especially forwards, display poor antagonist/agonist muscle-strength ratios with regard to their shoulder rotator muscles. ${ }^{[12]}$

Previous research has indicated a possible association between posture, isokinetic strength, scapular muscle recruitment patterns and injury among the general population and overhead athletes. ${ }^{[1]}$ However, there are few available profiles of rugby union players with regard to the aforementioned factors and the possible associations thereof with injury. The compilation of such profiles for uninjured players could, in theory, assist in the identification of rugby union players who are more likely to be at risk of future injury to the shoulder area. The aim of our study was, therefore, to profile rugby union players accordingly, to assist in the identification of possible musculoskeletal weaknesses.

Here we report on part of a larger study into the occurrence of shoulder injuries during the 2010 rugby union season at North-West University (NWU)-Puk Rugby Institute. The shoulder is the most injured body region in backline players at the institute. Previous work has implicated certain biomechanical and postural aspects, such as tight shoulder internal rotators and adductors, high body mass and kyphosis, as possible intrinsic risk factors for these shoulder injuries; however, the practical significance of these correlations was limited by small sample sizes. We subsequently performed a descriptive study to profile the thoracic posture, scapular muscle activation patterns and rotator cuff muscle isokinetic strength of right-hand-dominant semiprofessional rugby union players.

\section{Methods}

\section{Participants}

Ninety-five uninjured male rugby union players aged 17 - 31 years were recruited for the study. All participants were PUK U19 A/B, PUK U21 A/B (NWU-Puk Rugby Institute) or Leopards Rugby Union senior players (Provincial) based in the North West Province. All participants gave informed consent to participate in the study following an explanation of the test procedure and study protocols. The Ethics Committee of NWU approved the study (NWU-0004811A1). All participants were tested in the pre-season to ensure that they were uninjured during the test phase. Left-hand-dominant participants $(n=4)$ were excluded from the analysis.

\section{Measurements \\ Demographic information}

The stature of each participant was measured to the nearest $0.1 \mathrm{~cm}$ with a stadiometer (Seritex) using the stretch-stature method. Body mass was measured to the nearest $0.1 \mathrm{~kg}$ with an electronic weighing scale (Micro). Participants completed an information sheet surveying age, position of play, dominant side and previous injuries.

\section{Shoulder range of motion}

Biomechanical tests were performed according to a pro forma protocol compiled from various sources. Shoulder range of motion (ROM) was determined by the hand-behind-the-neck and -back tests. During both tests, participants stood in an upright position. In the hand-behind-the-neck test, participants were instructed to reach over their ipsilateral shoulder with one hand and place it as far down the spinal column as possible. The end-point of movement was marked (representing the most inferior point) with the shoulder in a position of external rotation (ER). Using the same technique, the contra-lateral hand was placed as far down the spinal column as possible, and the end-point was marked. The distance between the two marks was measured. The players were classified in terms of the discrepancies between the left- and right-shoulder ROMs: a difference $<1 \mathrm{~cm}$ was classified as ideal, differences of $1-3 \mathrm{~cm}$ were classified as non-ideal, and differences $>3 \mathrm{~cm}$ were classified as unsatisfactory. During the hand-behind-the-back test, the same principles were applied; however, participants were instructed to place their hands as high as possible on the spinal column (representing the most superior point), with the shoulder in a position of internal rotation (IR).

\section{Thoracic posture}

The New York Posture Test, designed for identifying 13 categories of deformities, ${ }^{[13]}$ was used for the evaluation and identification of possible postural deformities in the participants. Assessments were performed by capturing high-quality digital photographs of the lateral and posterior view of each participant. The camera was placed at a $90^{\circ}$ angle to the shoulders to ensure accurate calculation of angles. The photographs were analysed with Dartfish software (version 4.06.0; DARTFISH, Switzerland). A score of 5 (normal posture), 3 (slightly abnormal posture/moderate deviation) or 1 (abnormal posture/major deviation) was assigned to forward head, winged scapulae/round shoulders and kyphosis aspects of the postures. Uneven shoulders were measured by placing bright-yellow markers, $1 \mathrm{~cm}$ in diameter, on the posterior-lateral edges or acromial angles of the left and right acromions. Uneven shoulders were defined by the angle formed between the line connecting the inferior edges of the markers and a true horizontal line. To reduce the degree of subjectivity, New York Posture Test criteria were used to score uneven shoulders as follows: $5\left(0-2^{\circ}\right) ; 3\left(2.1-4.0^{\circ}\right)$ and $1\left(>4^{\circ}\right)$.

\section{Scapular muscle activation patterns}

EMG activities of the scapulothoracic muscles were registered by means of bilateral and simultaneous abduction of both arms in the scapular plane $\left(30^{\circ}\right.$ in front of the coronal plane).$^{[1]}$ The output of muscle activation was measured in microvolts $(\mathrm{mV})$. The firing sequence of the muscles was determined by measuring latency times $(\mathrm{ms})$. Consequently, the frequency (percentage of times) that a specific muscle group fired in a specific order was calculated. Accordingly, the muscles were classified in terms of firing sequence. Data were obtained with the Myotrace 400 Biofeedback system (Noraxon USA Inc.), which operates by means of a 4-channel transmitter that allows for simultaneous data collection from 4 strategically placed electrodes. EMG electrodes were attached unilaterally to the upper and lower trapezius, serratus anterior and infraspinatus muscles, respectively, in accordance with Surface ElectroMyoGraphy for the Non-Invasive 
Assessment of Muscles (SENIAM) guidelines. ${ }^{[14]}$ The overlying skin on the muscles was carefully prepared by abrading the outer epidermal layer and removing oil and dirt with alcohol pads. ${ }^{[15]}$ As only 4 channels were available to do the tests, the 4 muscles were measured unilaterally, after which the test was repeated on the contra-lateral side. The participants started the required movement with their arms resting next to their sides. Bilateral arm abduction in the scapular plane was performed to a point of $180^{\circ}$ of abduction, after which adduction was performed to the original starting point. The test was standardised for both sides by regulating the tempo of abduction and adduction. Participants performed the total abduction-adduction sequence in 7 seconds. No resistance was used or applied during the movement.

\section{Rotator cuff isokinetic muscle strength}

The torque/peak power and muscle agonist/antagonist ratios of the shoulder were tested with the Kin-Com $500 \mathrm{H}$ isokinetic dynamometer (Chattanooga, Tennessee) with torque/power expressed in Newton meters $(\mathrm{Nm})$. Torque scores are representative of the moment of force produced by muscle contraction for rotation around a joint. ${ }^{[12]}$ During shoulder IR and ER, the participant was seated and strapped to the seat. Testing was performed with the arms positioned along the scapular plane, at $90^{\circ}$ of abduction and with $90^{\circ}$ of elbow flexion. The contralateral arm was held static against the chest throughout the test and the feet were placed on a footrest. The shoulder axis of rotation was aligned with the dynamometer's axis of rotation. The 2 rotation points were connected with an imaginary line that runs from the dynamometer's axis of rotation, through the humerus, towards the acromion process. Each test started from the point of full ER. Participants warmed up using the Monark 881E Rehab Trainer (Monark, Sweden) for 3 min. Before the test commenced, each participant was informed about the test procedure. Three sub-maximal warm-up repetitions preceded the true test. Verbal encouragement was given during the test to ensure maximal torque output. The actual test consisted of a range of 6 concentric and eccentric maximal contractions. The maximal concentric and eccentric torque levels (in $\mathrm{Nm}$ ) of the shoulder-girdle complex were determined at speeds of $60 \% \mathrm{sec}$ for IR and ER. The above-mentioned values were used to calculate the different isokinetic ratios that were used to evaluate shoulder-muscle performance: the antagonist/agonist ratio and bilateral strength deficit ratio for concentric and eccentric contractions. Furthermore, the functional strength ratio was expressed as the eccentric ER torque production divided by the concentric IR torque production of a shoulder. This functional ratio appears to be relevant among overhead athletes, due to the fact that an increased activity of the external rotators is required to decelerate the humerus to centre the humeral head during a ballistic action. ${ }^{[16]}$ The dominant and non-dominant shoulders of each participant were measured.

\section{Statistical analysis}

SPSS software (version 17.0; IBM, New York) was used for statistical analyses. Descriptive statistics were performed to determine the characteristics of the participants as well as the profiles of the different variables. Frequencies and means with standard deviations (SDs) were calculated. Paired $t$-tests were performed to determine the differences between the measurements of dominant and non-dominant sides of the same individual. The level of significance was set at $p<0.05$.
Results

Participant characteristics (Table 1 ) indicated that $42 \%$ played rugby union as forwards. Statistically significant differences were found between forwards and backline players with regard to stature or height, weight, body mass index (BMI) and previous injuries to the shoulder joint. Twenty-eight per cent of the participants had suffered previous injuries to the shoulder, including previous surgery, dislocations or subluxations, and any injury that required the player to seek medical attention for intervention. The injuries could have been sustained at any stage, up until the end of the preceding season.

ROM tests were performed to determine the comparative flexibility of the shoulder internal and external rotators (hand-behind-the-neck and -back tests, respectively) (Table 2). Sixty-one per cent of the participants displayed non-ideal or unsatisfactory flexibility of their internal rotators when compared bilaterally. With regard to external rotator flexibility, upon bilateral comparison $84 \%$ of the participants were classified as non-ideal or unsatisfactory.

From the New York Posture Test ${ }^{[13]}$ used to evaluate thoracic posture (Fig. 1), more than half of the participants displayed a slightly abnormal or abnormal forward head position and a normal classification regarding a rounded back. The majority of the participants displayed normal posture with regard to uneven shoulders. Notably, $67 \%$ of the participants were classified as slightly abnormal or abnormal regarding their forward shoulder position.

In terms of the average firing order of muscle activation on the dominant side, the consensus sequence was: (i) serratus anterior, (ii) lower trapezius, (iii) infraspinatus and (iv) upper trapezius (Fig. 2; $x$-axis indicates the firing order, $y$-axis indicates the frequency of that order). The serratus anterior had the highest frequency for firing first (40\%) and the lower trapezius had the highest frequency for firing second (42\%). A similar firing order was observed on the

Table 1. Participant characteristics

\begin{tabular}{llll}
\hline & \multicolumn{3}{l}{ Mean $( \pm \mathbf{S D})$} \\
\cline { 2 - 4 } Variable & All & Forwards & Backs \\
\hline Age (years) & $20.8( \pm 2.9)$ & $20.8( \pm 2.7)$ & $20.7( \pm 3.0)$ \\
Stature $(\mathrm{cm})$ & $182.0( \pm 8.1)$ & $186.5( \pm 7.9)^{*}$ & $178.4( \pm 6.4)^{*}$ \\
Mass $(\mathrm{kg})$ & $91.5( \pm 15.1)$ & $103.3( \pm 13.3)^{*}$ & $82.2( \pm 8.6)^{*}$ \\
Previous injury (\%) & 27.5 & 40 & 17.6 \\
BMI $\left(\mathrm{kg} / \mathrm{m}^{2}\right)$ & $27.5( \pm 3.3)$ & $29.7( \pm 3.5)^{*}$ & $25.8( \pm 1.7)^{*}$ \\
& & & \\
SD = standard deviation; BMI $=$ body mass index. & & \\
*Significant difference $(p<0.05)$. & &
\end{tabular}

Table 2. Frequency of shoulder flexibility (non-dominant $\mathbf{v}$. dominant)

\begin{tabular}{llll}
\hline & $\begin{array}{c}\text { Ideal } \\
\text { ROM test }\end{array}$ & $\begin{array}{l}\text { Non-ideal } \\
\%\end{array}$ & $\begin{array}{l}\text { Unsatisfactory } \\
\%\end{array}$ \\
\hline Hand-behind-the-neck & 39.3 & 42.7 & 18.0 \\
Hand-behind-the-back & 15.7 & 23.6 & 60.7 \\
ROM = range of motion. & & &
\end{tabular}




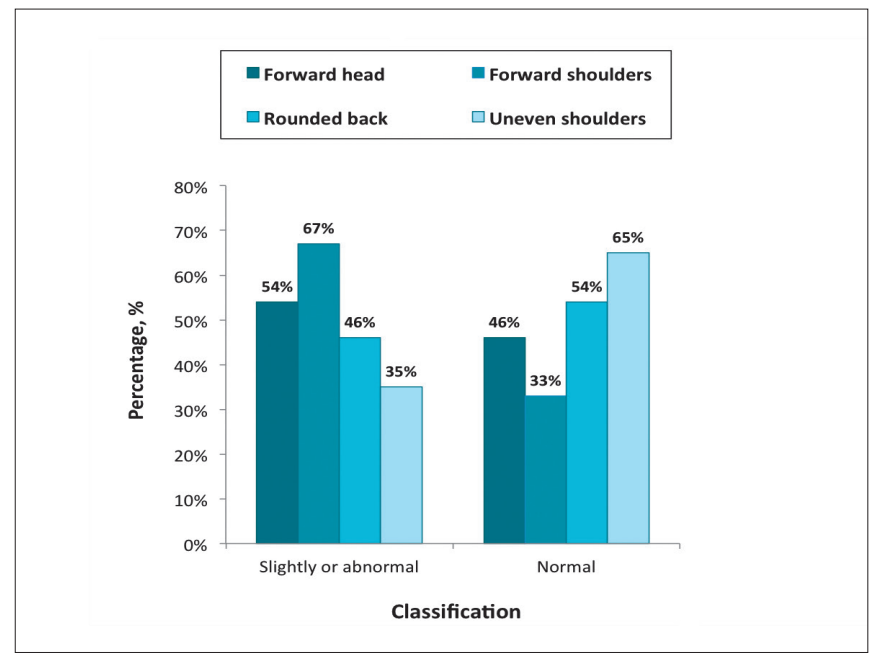

Fig. 1. Thoracic postural profile of participants according to the New York Posture Test. ${ }^{[20]}$

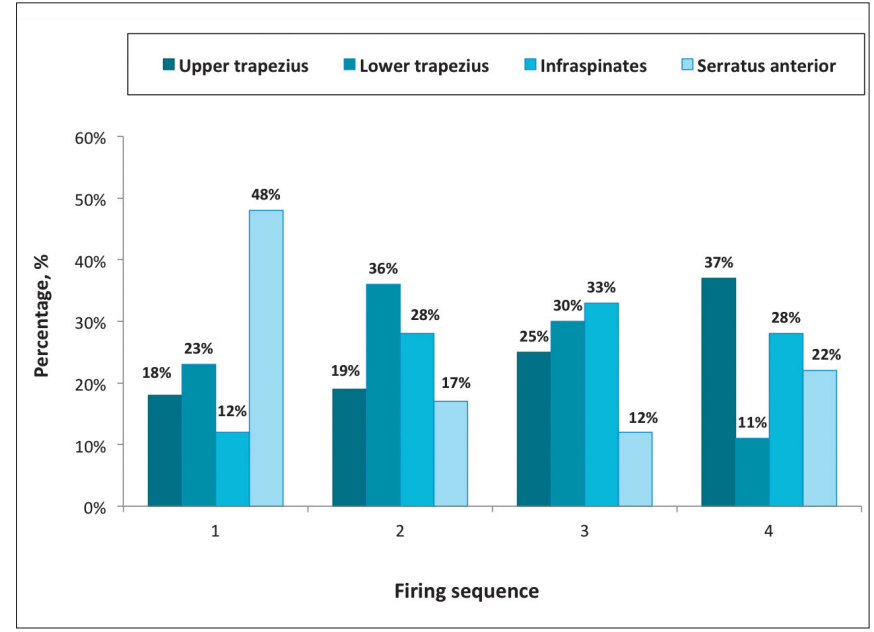

Fig. 2. Percentage of times that the dominant upper trapezius, lower trapezius, infraspinatus and serratus anterior muscles fired in a specific order during abduction in the scapular plane.

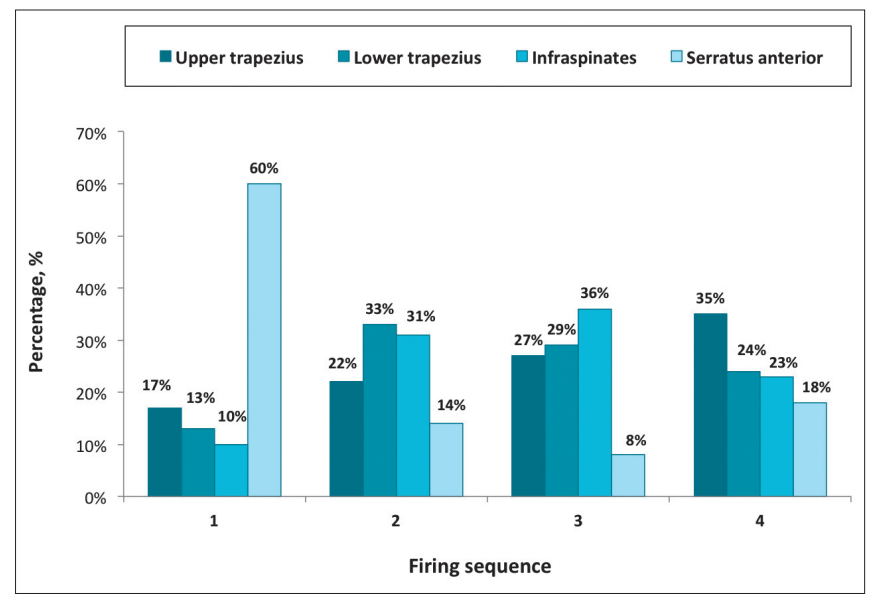

Fig. 3. Percentage of times that the non-dominant upper trapezius, lower trapezius, infraspinatus and serratus anterior muscles fired in a specific order during abduction in the scapular plane. non-dominant side, despite the fact that different frequencies were observed (Fig. 3).

The results of testing the isokinetic shoulder strengths with the dynamometer (Table 3) were that the antagonist/agonist ratio regarding concentric shoulder rotation of the non-dominant shoulder was slightly lower than what is regarded as acceptable (64\%). The corresponding ratio for the dominant shoulder was even lower. A statistically significant difference was observed between the values for the right and left sides. A statistically significant difference was also found between the antagonist/agonist ratio regarding eccentric shoulder rotation of the non-dominant shoulder and the antagonist/ agonist ratio regarding eccentric shoulder rotation of the dominant shoulder. The bilateral deficit during concentric IR indicated that the participants' non-dominant shoulders were generally stronger than their dominant shoulders during IR. With regard to concentric ER, the participants' shoulders also appeared to be stronger on the nondominant side. The bilateral deficit during eccentric IR shows that this right-dominant group was stronger on the dominant side. When one considers the ER component, it seems that there is parity between the average strength of the dominant and non-dominant shoulders.

Table 3. IR and ER isokinetic muscle-strength ratios

\begin{tabular}{ll}
\hline Muscle movement & Mean $( \pm$ SD $)$ \\
\hline CND ER/IR & $64.14( \pm 14.41)^{*}$ \\
CD ER/IR & $54.55( \pm 10.18)^{*}$ \\
END ER/IR & $67.24( \pm 11.62)^{\star}$ \\
ED ER/IR & $61.52( \pm 9.73)^{*}$ \\
ND/DcIR & $90.65( \pm 12.82)$ \\
ND/DcER & $106.17( \pm 19.50)$ \\
ND/DeIR & $92.85( \pm 11.74)$ \\
ND/DeER & $101.03( \pm 15.68)$
\end{tabular}

$\mathrm{IR}=$ internal rotation; $\mathrm{ER}=$ external rotation; $\mathrm{SD}=$ standard deviation; $\mathrm{CND}$ ER/IR $=$ concentric non-dominant external rotation/internal rotation; $\mathrm{CD}$ ER/IR = concentric dominant external rotation/internal rotation; END ER/IR = eccentric non-dominant external rotation/internal rotation; $\mathrm{ED} \mathrm{ER} / \mathrm{IR}=$ eccentric dominant external rotation/internal rotation; $\mathrm{ND} / \mathrm{DcIR}=$ non-dominant/dominant concentric internal rotation; ND/DcER = non-dominant/dominant concentric external rotation; ND/DeIR = non-dominant/dominant eccentric internal rotation; ND/ DeER $=$ non-dominant $/$ dominant eccentric external rotation

*Significant difference $(p<0.05)$

\section{Discussion}

The main objective of this study was to profile semi-professional rugby union players in terms of thoracic posture, scapular muscle activation patterns and rotator cuff isokinetic muscle strength. The results indicated that the majority of the players of the Leopards Rugby Union and NWU-Puk Rugby Institute had less than ideal or unsatisfactory flexibility of their external shoulder rotators when the left and right shoulders were compared. Testing the flexibility of the shoulder internal rotators indicated that only a small percentage of the players had ideal flexibility when their left and right shoulders were compared. This supports the findings of a previous study ${ }^{[17]}$ of a diminished glenohumeral rotation range among professional rugby players in comparison with a control group. Another study also reported deficiencies in rugby players' $\mathrm{ROM}$, possibly attributed to 
age, playing position, body mass index (BMI) or a history of injury. It was previously found that age could be a risk factor for decreased flexibility of shoulder rotators. ${ }^{[17]}$ This could be related to the ageing of the glenohumeral soft tissue, which may be accelerated by the training and injuries associated with rugby. ${ }^{[17]}$ Deficiencies in ROM can be regarded as a risk factor for future injuries. The areas affected by decreased ROM are obviously less mobile. Consequently, the joint's supporting structures are dynamically loaded and susceptible to intrinsic injury.

The results further indicate a higher prevalence of abnormal thoracic posture than reported in the literature for a non-sporting population. In the latter, $66 \%$ of subjects had a forward head, $60 \%$ had thoracic spine kyphosis, and $38 \%$ had rounded shoulders. ${ }^{[2]}$ This higher prevalence could have been attributed to the poor flexibility of certain anatomical structures, incorrect strength training, or incorrect conditioning techniques applied among the players. To our knowledge, no research has been done on incorrect training techniques among rugby union players and the association thereof with poor posture. However, it has been found that decreased resting length of the pectoralis minor muscle could have a negative influence on scapular kinematics. Therefore, a strength programme where there is an imbalance between pectoralis major strengthening (too much) $\mathrm{v}$. latissimus dorsi strengthening (insufficient), may contribute towards a shortened pectoralis major muscle. This may also be exaggerated by insufficient stretching of the pectoralis major muscle. This could result in a scenario where posture, and consequently scapular kinematics, may be negatively influenced, ultimately increasing the risk of injury. However, there is an argument that certain postural deviations such as abducted scapulae and rounded shoulder posture may be advantageous for contact sport athletes. The theory is that this posture allows the athlete to assume a tuck or covered-up position quickly before making contact with defending players. However, this seems to be a matter of opinion, and no sufficient scientific data exist to confirm this theory. Some believe that a link exists between posture and ROM. ROM loss may be directly attributed to changes in thoracic posture. Such changes may cause a reduction in the sub-acromial space, which may cause impingement of supra-humeral soft tissue and subsequently reduce the overall ROM and increase the likelihood of injury. Theoretically, the high percentage of postural deformities within this group, given the high physical demands placed on rugby union players, could make this group susceptible to future shoulder injury. Poor posture, therefore, not only influences ROM but also impedes optimal scapular kinematics.

Knowledge of scapular muscle timing patterns is vitally important in terms of our understanding of the behaviour of the joint system, particularly under demanding circumstances. It is relevant to profile rugby union players with regard to these patterns. EMG analyses present information on the sequence in which the scapular stabilisers fire during shoulder movement along a scapular plane. In this study the consensus sequence was: (i) serratus anterior, (ii) lower trapezius, (iii) infraspinatus, and (iv) upper trapezius. Previous research regarding EMG analysis of rugby players' scapulothoracic muscles is limited, but the sequence of muscle activation patterns in selected shoulder girdle muscles during a front-on tackle in asymptomatic rugby players has previously been investigated. ${ }^{[5]}$ The authors found a consistently earlier activation of serratus anterior compared with the pectoralis major, biceps brachii, latissimus dorsi and infraspinatus. In accordance with our study, even though different movements were measured, the results also indicated that the serratus anterior was the first muscle to fire before the other muscles tested. The influence of a superior labral tear from anterior to posterior (SLAP lesion) on the onset of EMG activity in shoulder muscles during a front-on tackle among professional rugby union players has also been investigated. ${ }^{[18]}$ Again, results indicated that the onset of serratus anterior muscle activity occurred significantly earlier than the other muscles examined. This was seen, despite a trend towards a delay in activation time of all the other muscles within the injured group. ${ }^{[19]}$ It is obvious that serratus anterior plays a significant role in the initial stabilisation of the scapulothoracic joint in a simulated tackle situation. It is postulated that a delay in the activity of serratus anterior, and the subsequent impairment in scapular control, would allow the humeral head to translate anteriorly and superiorly when the humerus reached an abducted position in the tackle situation. This could ultimately have a detrimental effect on the dynamic stability of the glenohumeral joint. ${ }^{[19]}$

During our study the most frequent firing order for the trapezius muscles was the lower trapezius second and the upper trapezius fourth. It has been postulated that an early activation of the stabilising muscles at the proximal scapulothoracic joint is important for maintaining proper scapulothoracic stability throughout glenohumeral movement, and that the correct sequence of these muscles' activity is critical for normal scapular kinematics. ${ }^{[1]}$ The muscles that aid the serratus anterior in providing dynamic stability to the scapula, provide a force by coupling around the scapula. ${ }^{[8]}$ The infraspinatus plays a role in posterior glenohumeral joint stability, but its ability to provide early support to this joint is apparently impaired by injury. It has been shown that the infraspinatus activates significantly earlier than the pectoralis major and latissimus dorsi during a simulated tackle situation, but that this earlier activation is not seen among injured players. This may be indicative of a failure of the local control system that could possibly lead to increased stress on the shoulder support structures. All the muscles that were tested by means of EMG during this study have important functions regarding the normal shoulder function of rugby players, and it has been shown that the correct timing of the activation of these muscles is significant. ${ }^{[1,8,19]}$ Altered muscle activation patterns could contribute towards scapular dyskinesia or indicate underlying injury, but normative data regarding correct muscle activation patterns could possibly aid in identifying potential weaknesses among scapular stabilisers before injury occurs.

During this particular study, the antagonist/agonist ratio regarding concentric isokinetic shoulder rotation of the right shoulder was only $55 \%$, while that of the left shoulder was $64 \%$. These findings are comparable with those of another study ${ }^{[17]}$ where a concentric external/internal rotator muscle ratio of $64 \%$ and $56 \%$ was reported for rugby backline and forward players, respectively. In both studies, the suggested antagonist/agonist muscle-strength ratio of $65 \%{ }^{[16]}$ was not found. This could be relevant due to the fact that muscle imbalances around a specific joint may increase the risk of injury to athletes. ${ }^{[16]}$ The statistically significant difference between the players' dominant and non-dominant antagonist/agonist ratios in the current study is also noticeable. It is unclear why the dominant shoulders generally tended to have weak antagonist/agonist ratios. The tackle is the phase of play in which most game injury events occur. ${ }^{[20]}$ If rugby players 
generally tend to tackle with their dominant shoulder, the possibility could exist that training and tackling over a period of time may have a detrimental effect on the soft-tissue supporting structures around the shoulder joint. This may impair normal functioning and, even though players may not perceive being injured, the wear and tear may be manifested in inadequate shoulder-strength ratios. Another area of interest lies in the fact that bilateral comparisons displayed that the participants had, on average, stronger concentric IR and ER strength on the non-dominant than the dominant side. Despite the fact that varied results have been found regarding dominant v. non-dominant shoulder strengths for overhead athletes, it has generally been found that the dominant side is as strong as, ${ }^{[21]}$ or stronger than, ${ }^{[22]}$ the nondominant side. The findings of our study are therefore contrary to those of previous research and may be indicative of the weakness of our participants' dominant concentric IR and ER strength.

\section{Study limitations}

Certain limitations of our study should be acknowledged. We employed the New York Posture Test due to a lack of objective posturemeasurement techniques; however, this test was initially designed to classify adolescents and is therefore not ideal for our group of participants, of whom a large proportion were beyond the adolescent stage (range 17 - 31 years). Secondly, regarding ROM measurement, a more scientific and objective method is required than employed in this study. Lastly, with regard to the measurement of scapular muscle activation patterns, the information would be more relevant if the muscles were to be tested during more functional, rugby-applicable movements, such as tackling, for instance.

\section{Conclusion}

A large percentage of the participants in our study displayed non-ideal or unsatisfactory flexibility of the shoulder internal rotators. More than two-thirds displayed forward shoulders and more than half of the participants had unsatisfactory or non-ideal head positions. These are all indicative of a kyphotic posture. The firing sequence in abduction in a scapular plane and in both shoulders was: (i) serratus anterior, (ii) lower trapezius, (iii) infraspinatus, and (iv) upper trapezius. As the participants were uninjured, this firing order may indicate the normal sequence of rugby players' scapular stabilisers during abduction in a scapular plane. It appears that the firing order of serratus anterior, prior to those of the other muscles studied, may be important for rugby players to maintain healthy shoulder function. The isokinetic shouldermuscle strength and ratios indicated a possible deficiency with regard to ER strength in the dominant shoulder. This is possibly manifested in an unsatisfactory antagonist/agonist shoulder rotation ratio. The profile of the thoracic posture of the participants presents an image of a kyphotic rugby player with an inappropriate ROM. This, in combination with an apparent weakness of right shoulder external rotator strength among the players, could have an impact on the prevalence of future injury from a biomechanical point of view, especially in the game of rugby with ever-increasing physical demands placed on players. By identifying these apparent musculoskeletal weaknesses, it may be possible to rectify them pro-actively with prehabilitation.
Conflict of interest. The authors have no conflicts of interest to declare.

\section{References}

1. Wadsworth D, Bullock-Saxton J. Recruitment patterns of the scapular rotator muscles in freestyle swimmers with subacromial impingement. Int J Sports Med 1997;18(8):618-624. [http://dx.doi.org/10.1055/s-2007-972692]

2. Brooks J, Fuller C, Kemp S, Reddin D. Epidemiology of injuries in English professional rugby union: Part 1, Match injuries. Br J Sports Med 2005;39(10):757766. [http://dx.doi.org/10.1136/bjsm.2005.018135]

3. Funk L, Snow M. SLAP tears of the glenoid labrum in contact athletes. Clin J Sport Med 2007;17(1):1-4. [http://dx.doi.org/10.1097/JSM.0b013e31802ede87]

4. Griegel-Morris P, Larson K, Mueller-Klaus K, Otis C. Incidence of common postural abnormalities in the cervical, shoulder, and thoracic regions and their associations with pain in two age groups of healthy subjects. Phys Ther 1992;72(6):426-431.

5. Greenfield B, Catlin P, Coats P, Green E, McDonald J, North C. Posture in patients with shoulder overuse injuries and healthy individuals. J Orthop Sports Phys Ther 1995;21(5):287-294.

6. David G, Magarey M, Jones M, Dvir Z, Turker K, Sharpe M. EMG and strength correlates of selected shoulder muscles during rotations of the glenohumeral joint. Clin Biomech 2000;15(2):95-102. [http://dx.doi.org/10.1016/S0268-0033(99)000522]

7. Kibler W. The role of the scapula in athletic shoulder function. Am J Sports Med 1998;26(2):325-337.

8. Cools A, Witvrouw E, Declercq G, Danneels L, Cambier D. Scapular muscle recruitment patterns: Trapezius muscle latency with and without impingement symptoms. Am J Sports Med 2003;31(4):542-549.

9. Herrington L, Horsley I. Electromyographic analysis of selected shoulder muscles during a rugby football tackle. Sports Med Arthroscopy Rehab Ther Tech 2009;1:10. [http://dx.doi.org/10.1186/1758-2555-1-10]

10. Wilk K, Obma P, Simpson C, Cain E, Dugas J, Andrews J. Shoulder injuries in the overhead athlete. J Orthop Sports Phys Ther 2009;39(2):38-54. [http://dx.doi. org/10.2519/jospt.2009.2929]

11. Le Roux J, Codine P, Thomas E, et al. Isokinetic evaluation of rotational strength in normal shoulders and shoulders with impingement syndrome. Clin Orthop 1994;304:109-115.

12. Coetzee F, McDonald T, Kilian J. A comparison of maximal torque levels of the different planes of movement of the shoulder-girdle complex for different types of sports. S Afr J Res Sport Phys Educ Rec 2002;24(1):17-27.

13. Magee D. Orthopaedic physical assessment. 4th ed. Philadelphia: Saunders, 2002:1020.

14. Hermens HJ, Freriks B, Merletti R, et al. [SENIAM 8] European Recommendations for Surface ElectroMyoGraphy; deliverable of the SENIAM project. Nijmegen, The Netherlands: Roessingh Research and Development, 1999.

15. Hunter A, St Claire Gibson A, Lambert M, Nobbs L, Noakes T. Effects of supramaximal exercise on the electromyographic signal. Br J Sports Med 2003;37(4):296-299. [http://dx.doi.org/10.1136/bjsm.37.4.296]

16. Bak K, Magnusson S. Shoulder strength and range of motion in symptomatic and pain free elite swimmers. Am J Sports Med 1997;25(4):454-459. [http://dx.doi. org/10.1177/036354659702500407]

17. Fernández J, Aravena R, Verdugo R, Echeñique S, Fei to M, Rex F. Glenohumeral rotation range deficit in professional rugby players: A cross sectional study. Int J Sport Sci 2011;7(22):39-47. [http://dx.doi.org/10.5232/ricyde2011.02204]

18. Edouard P, Frize N, Calmels P, Samozino P, Garet M, Degache F. Influence of rugby practice on shoulder internal and external rotators strength. Inter J Sports Med 2009;30(12):863-867. [http://dx.doi.org/10.1055/s-0029-1237391]

19. Horsley I, Herrington L, Rolf C. Does a SLAP lesion affect shoulder muscle recruitment as measured by EMG activity during a rugby tackle? J Orthop Surg Res 2010;5(1):1-10. [http://dx.doi.org/10.1186/1749-799X-5-12]

20. Fuller C, Brooks J, Cancea R, Hall J, Kemp S. Contact events in rugby union and their propensity to cause injury. Br J Sports Med 2007;41(12):862-867. [http:// dx.doi.org/10.1136/bjsm.2007.037499]

21. Mikesky A, Edwards J, Wigglesworth J, Kunkel S. Eccentric and concentric strength of the shoulder and arm musculature in collegiate baseball pitchers. Am J Sports Med 1995;23(5):638-642. [http://dx.doi.org/10.1177/036354659502300520]

22. Chandler T, Kibler W, Stracener E, Ziegler A, Pace B. Shoulder strength, power, and endurance in college tennis players. Am J Sports Med 1992;20(4):455-458. [http:// dx.doi.org/10.1177/036354659202000416] 